\title{
Application of Cox's Proportional Hazard Model and Construction of Life Table for Under-Five
}

\author{
Salam Shantikumar Singh ${ }^{*}$
}

Department of Statistics, Pachhunga University College, Aizawl-Mizoram, India

\begin{abstract}
A primary data of 836 eligible women in the age group of 15-49 years is used to determine the causal effects of covariates on under-five mortality. The eight covariates viz., number of family members (NHM), type of toilet facility (TTF), total children ever born (TCB), parity (PAR), duration of breastfeeding (DBF), use contraceptive (CMT), DPT and ideal number of girl (ING) are considered as covariates of the study. By applying Cox's regression analysis, six covariates viz., TTF, NHM, CMT, DBF, DPT and ING have substantially and significantly effect on under-five mortality. Further, a life table of under-five children under study is constructed using the estimate of survival function obtained from Cox's regression model.
\end{abstract}

Keywords: Under-five, Covariates, Cox's regression, hazard function and life table.

\section{INTRODUCTION}

The first five years of life are the most crucial to the physical and intellectual development of children and can determine their potential to learn and thrive for a life time. That is why it is specifically stated as one of the goals of the millennium development goals (MDGs) to reduce child mortality by two-thirds by 2015 . Although there has been a substantial reduction in infant and child mortality rates in most developing countries in the recent past, it still remains a major public health issue in South Asian countries particularly in India.

Mortality and its converse indicator, longevity or life expectancy are among the most important measures of well-being and development in developing countries. Since child mortality has an overwhelming influence on life expectancy, it is important to analyze the determinants of child mortality in India and particularly in the state of Manipur. Moreover, child mortality indicates the health status of not only child but also the health status of mothers as well as society as a whole. The child mortality has received a new momentum of the study since there is a strong association ship between mortality and fertility as high mortality corresponds high fertility and vice-versa. Thus, the study of especially on child has as immense contribution towards the regulation of population growth and enhancing the health status of the society.

The general medical definition distinguishes mortality of a child with respect to the child age: death within the first week of life is included with prenatal

*Address correspondence to this author at the Department of Statistics, Pachhunga University College, Aizawl, Mizoram, PIN NO. 796001, India; Tel: (+91)8732843655; E-mail: shantikumarsalam@yahoo.co.in mortality (which also includes late foetal mortality) and death within the first month is referred to as neonatal mortality, and death within one year is referred to as infant mortality. The death under five is referred to as child mortality (WHO, (2005) [1]). Since peri and neonatal mortality is heavily influenced by prematurity, fatal genetic conditions of the foetus, and problems associated with delivery. The mortality after first month, which is mostly related to socio-economic and health conditions of the household. It is possible to analysis the determinants of child mortality at various levels of causality (Mosley \& Chen (1984) [2]). The biomedical and epidemiological literature typically focuses on the immediate determinants of child mortality, in particular the impact of various diseases and weakened resistance. In contrast, socio-economic, environment \& sanitation, medical and health care, demographic, exposure to mass media, etc., are usually focused on underlying determinants of child mortality that make children more vulnerable to the attack of various diseases. Moreover, the child mortality rates vary from countries to countries and even within the country also it is varied in region to region and state to state. In developed countries, the main factor influencing on child mortality is demographic factors whereas socioeconomic, health care, etc., are main factors influencing on child mortality in developing countries. Thus, the study of child mortality is different from country to country and region to region.

\subsection{Review of Literature}

From the past research findings, it is known that parity (birth order) is associated with infant and child mortality. Hobcraft et al. (1985) [3] indicate that beyond the first year of the morality of first born children is on average slightly lower than for children of birth orders 2 
or 3. As suggested by Majumder et al. (1997) [4], birth order has highly significant effect on mortality during infancy but it seems to be invisible during children age of between 1 to 5 years. Moreover, in a study of Pandey et al. (1998) [5], demographic characteristics have substantial adjusted effects on mortality before age five. The unadjusted effects are not very different from the adjusted effects except in the case of birth order and mother's age at childbirth meaning that these two demographic factors have highly and substantially influenced on under5 mortality. But, adjusted neonatal mortality decreases with increasing birth order, whereas adjusted post-neonatal and child mortality increase with increasing birth order and the same finding is also observed by Biswas et al. (2000) [6]. As suggested by Syamala (2004) [7], the risk of dying during the first year of life is higher among children of lower and higher order births. The proportion of children surviving in the first year of life is lower among the first order births and it improves up to fourth order births and then declines for the births of order five and above. In both the cases, the pattern of infant mortality by age of the mother at the time of delivery as well by birth order exhibited a $U$ shaped relationship. Balhotra \& Soest (2005) [8] also report the same finding of the relationship between neonatal mortality and birth order exhibited U-shaped. An interesting finding from India's NFHS-3 report [9] highlights that the first births are more likely to be delivered in an institution than births at higher birth orders and hence first birth children are likely less chance of death than higher birth order due to unavailability of health care facility.

Fertility is the actual number of child bearing while fecundity is the capacity of bearing children. As a measure, "fertility rate" is the number of children born per couple, person or population. The relation between fertility and child mortality is at the core of historical and modern demographic transitions.

Some characteristics of children are related to mother's fertility behaviour, such as mother's age at childbirth, child's birth order, and previous and following birth intervals. These characteristics are known to affect neonatal, postneonatal, infant, and child mortality in developing countries (Hobcraft et al. (1985) [3]). First-born children and children of high birth orders are known to experience higher mortality than children of birth orders two to four. Children born to women under age 20 and over age 35 are known to have higher mortality than those born to mothers' age 20-34, most likely because a woman's physical condition is most favorable to childbearing during her twenties and early thirties. Infant and child mortality in India, a report of NFHS-2 (1998) [5], indicates that a decline in fertility, by reducing the proportion of higher-order births, will tend to lower the overall level of child mortality. Further, Bhuyan (2000) [10] agreed the above findings and suggests that the upward trend in child mortality is significantly and positively associated with fertility, thus higher fertility levels are associated with higher probabilities of child deaths.

As indicated by Rutstien (2000) [11], the fertility behaviour is represented by the demographic characteristics like birth order, mother's age at birth, and length of birth intervals. An increase in the percentage of births that are of first birth order was associated with higher mortality rates among postneonatal infants and toddlers but not with changes in other mortality rates. An increase in the percentage of births of fourth or higher order was associated with a decrease in mortality for all mortality rates. This finding is also in agreement with the finding of child mortality in rural India reflected in discussion paper of World Bank (2004) [12] and Adair (2004) [13].

NFHS-3 of India (2008) [14] reports that the component of fertility i.e., mother's age at birth, birth order, and the interval between births have a strong influence on infant and child mortality and suggests parents can increase the chances of their children's survival by controlling these proximate determinants.

The impact of breastfeeding on the survival status of child may be considered in two ways i.e., its effect to the mother and to the child. In the report "Infant and child mortality in the third world" (1983) [15], it has been observed that greater survivorship, and therefore lower infant mortality, is associated with the breastfeeding, absence of reproductive losses and mother's educational level. In the finding of Palloni \& Millman (1985) [16], there is little doubt that breastfeeding effects are important within the first year of life. Without considering the first month, there is a significantly uncovered significant mortality reduction attributable to breastfeeding. However, there is also a strong evidence supporting the nation that those effects were over time and are much more important among subgroups which are socially and economically deprived; not only do countries experiencing higher mortality rates show the most important contributions of breastfeeding, but the corresponding effects are exposed to higher levels of resource scarcity. In their study, Fauveau et al. (1990) [17] and Sandiford et al. (1991) [18] observe important causes of death among 
infants aged 1 to 5 months are severe malnutrition usually due to lack of breastfeeding. A similar finding is reported in India by Pandey et al. (1998) [5] and Claeson et al. (1999) [19]. And it is also reported by Bhuyan (2000) [10] that duration of breastfeeding and age of mother at marriage have influences in reducing mortality level of children. In a study of Rutstein (2000) [10], suggests that an increase in the duration of breastfeeding is associated with a fall in post-neonatal mortality. A rise in the percentage of children aged 7-9 months who were both breastfed and getting solid foods is associated with decrease in both post natal and infant mortality rates. Biswas et al. (2000) [6] also reported in their study on impact of some biosocial variables on infant and child mortality that breastfeeding appeared to be prime factor influencing infant, second year (12-23 months) and early child (2459 months). As highlighted by Griffiths et al. (2001) [20] the magnitude of the association is greatest for breastfeeding behaviour and early child mortality.

Mahy (2003) [21] indicates that the vertical transmission of HIV occurs in approximately 32 percent of births to HIV infected mothers in countries where breastfeeding is prevalent and it will directly affect on childhood mortality.

Access to a flush or pit toilet is potentially a very important determinant of infant and child mortality in developing countries. Children in households that lack such access could have higher exposure than other children to diseases such as tetanus and digestive disorders.

In a related study of Roth \& Kurup (1989) [22] suggests that good public sanitation systems may constitute a more important preventive aspect of child mortality. In the latter study of Kabir \& Amin (1993) [23] in Bangladesh also highlights that the households with sanitary latrines have low risks of child mortality. The similar finding is reported by Pandey et al. (1998) [5] that access to a flush or pit toilet households have substantial and often statistically significant adjusted effects on infant and child mortality. The adjusted effect on mortality of household access to a flush or pit toilet is strongest for the neonatal period and becomes weaker at later ages.

On the other hand, Baker (1999) [24] in his study on differential in child mortality in Malawi, in contrast to the above findings observes that access of pit latrine does not have a significant effect on child mortality in the country. The same finding is witnessed by Rutstein (2000) [11] in developing countries.
As highlighted by Klaauw \& Wang (2004) [12], access to toilet facility can reduce under-five mortality rate significantly in rural areas of India as a whole. In urban Kenya, access to modern sanitation facilities (flush toilets) reduces diarrhea prevalence in urban areas and ultimately reduce the child mortality, Mutunga (2004) [25]. In a study of Balk et al. (2005) [26], the principal component analysis is used to combine the correlated variables which influence on mortality. From their analysis it is found the mortality is correlated positively with the complete lack of toilet facilities and negatively with access to flush toilets.

It is also suggested by Vos et al. (2005) [27] that the availability of better sanitation will decrease the probability of infant death since better sanitation and drinking water access of the household should positively improve hygienic and health conditions for all members.

Babies are born with protection against certain diseases because antibodies from their mothers were passed to them through the placenta. After birth, breastfeeding babies get the continued benefits of additional antibodies in breast milk. But in both cases, the protection is temporary. Immunization (vaccination) is a way of creating immunity to certain diseases by using small amounts of a killed or weakened microorganism that causes the particular disease.

Children are considered fully immunized if they receive one BCG injection to protect against tuberculosis, three doses each of diphtheria, pertussis and tetanus (DPT) and polio vaccines, and one measles vaccine. In India, according to NFHS-3 report (2008) [14], only 44 percent of children age 12-23 months are fully vaccinated, and 5 percent have not received any vaccinations.

In a study of child mortality levels and patterns from Southern Sudan by Roth \& Kurup (1989) [22], it is witnessed that the increase in child survival may be reflected by the control and/or eradication of communicable disease by modern medical technology. The maternal education and immunization of children are the most significant determinants of child survival. According to Kabir \& Amin (1993) [23], health interventions such as immunization programmes might have had an effect on lowering infant and child mortality in Bangladesh.

Claeson et al. (1999 \& 2000) [19, 28] in their study based on NFHS-2 data indicate a positive relationship 
between coverage of key child health interventions (such as ORT, ARI, and immunization rates) and the reduction in under-five mortality. Effective interventions could result in a rapid reduction in the perinatal and neonatal mortality rates and therefore in the overall IMR (infant mortality rate) and U5M (under5 mortality). A study of Rutstein (2000) [11] on factors associated with trends in infant and child mortality in developing countries during the 1990s suggests that a change in the percentage vaccinated during pregnancy with tetanus toxoid did not explain the change in mortality for children aged less than 1 year. An increase in the percentage of children vaccinated against measles was associated with declines in infant mortality and with mortality at ages greater than 1 year. An increase in the percentage of children receiving medical attention for diarrhoea, acute respiratory illness and fever declines in child mortality.

In a study of Vos et al. (2005) [27], coverage of immunization is also very significantly related to lower prevalence of infant mortality. For each $1 \%$ increases in the coverage of immunization, infant mortality goes down by about $1.1 \%$. The effectiveness of this preventive action stands out. The simulations analysis also show that reaching full coverage of the immunization program or the expansion of the free maternity program targeted at the poor by themselves are not sufficient to reach the MDG targets for infant mortality. The expansion of the immunization program would reduce infant mortality to 20.1 per 1,000 live births by 2015. As the immunization program is universal and initial coverage does not differ much across population groups, this policy would reduce infant mortality for all, but would not narrow differences between poor and non-poor or indigenous and non-indigenous.

The rationale of the study is initiated empirically to analyze the effects of some important determinants which are expected to cause the child mortality. Further, a life table of children is to be constructed and it will visualize the mortality pattern at different stages of their life.

\section{METHOD}

The study design is cross sectional with survey period started from $1^{\text {st }}$ May, 2008 to $30^{\text {th }}$ April, 2009 in four districts of Manipur, India. A sample of 836 eligible women (age 15-49 years) have been selected by using two stage sampling with proportionately allocated to districts and villages. The Cox's proportional hazard regression analysis (Cox, 1972 [29]) is used to analyze the data. The effects of covariates on under-five are measured by using relative risk of each covariate and a life table of under-five children is constructed after estimating the survival function.

The survival time of a child is considered as response variable, and it is considered with respect to reference period. The children who live start within the reference period are taken into consideration. The

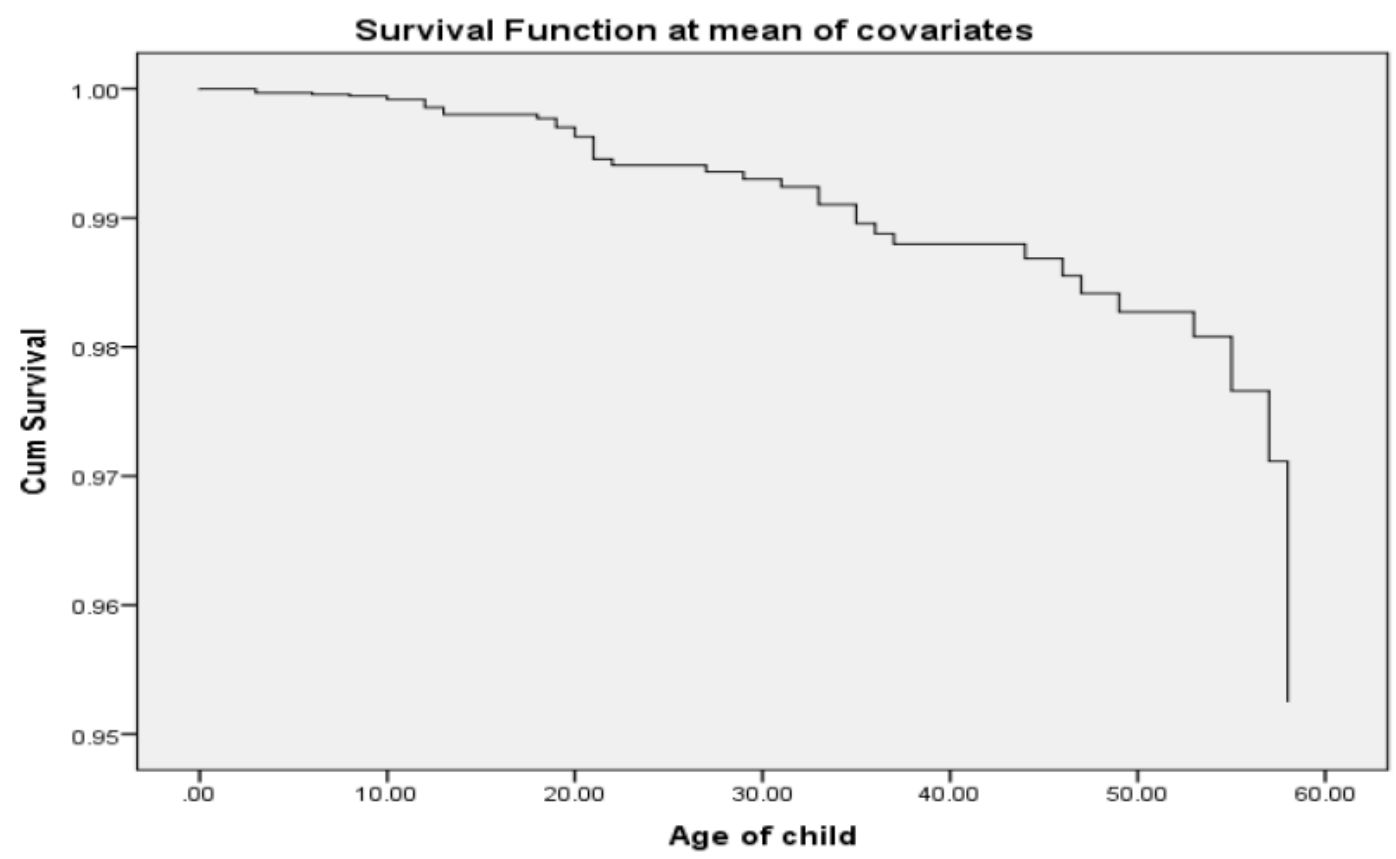

Figure 1: Survival function at the mean of covariates. 


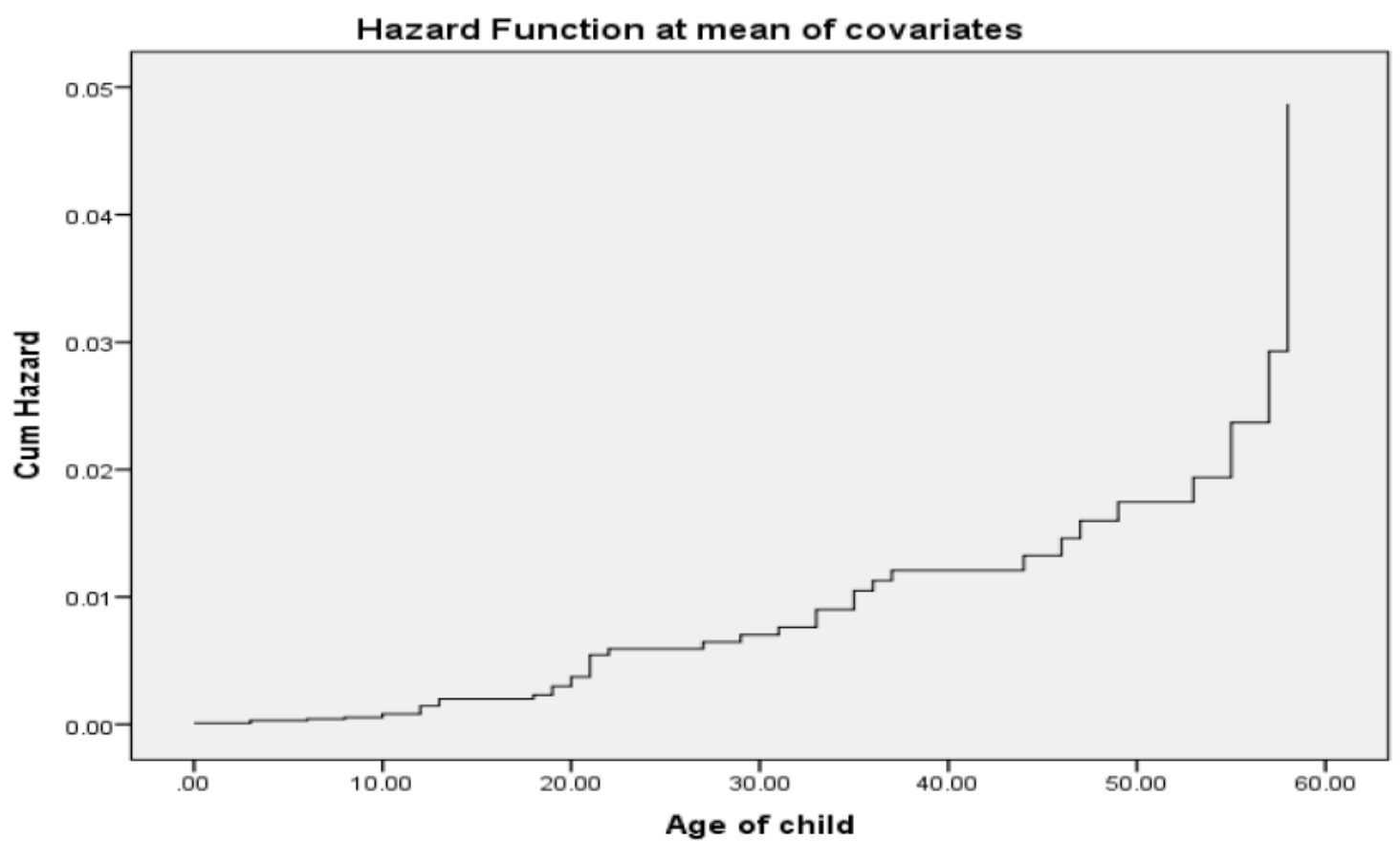

Figure 2: Hazard function at mean of covariates.

children died within the reference period are taken as uncensored cases the children alive in that period are censored cases. To identify whether a case is censored or not, an indicator variable called survival states of child (SSC) is assigned as 1 , if the child is death (event occur) in the reference period and 0 , otherwise (alive or censoring). Along with these, 8 covariates are taken into account such as type of toilet facility (TTF), number of family members (NHM), number of ever born children (TCB), use contraceptive (CMT), duration of breastfeeding (DBF), ideal number of girls (ING), DPT and parity (PAR). Again, the Cox's regression by stepwise method (Forward) is proposed to selection the best set of covariates to be included in the model.

The main purpose of this analysis is to obtain the values of the survivorship function $\bar{S}(t)$ at the mean values of the covariates. With these values, the survivorship function $S(t)$ can be estimated and then constructed the life table of under-five.

\section{RESULTS AND DISCUSSION}

The Cox's proportional hazard regression model is fitted to the data along with 8 covariates. The purposeful selection of variables and fix for a best subset of the covariates out of these 8 covariates has been conducted by stepwise method (Wald's forward) with $p$-value 0.05 for entry level of a covariate in the model and 0.10 for deletion level of a covariate in the model. For assessing the best fit of the model particularly model coefficients, overall model and goodness of fit are conducted by Wald's test, likelihood ratio test and score test. From this analysis, further, interpretation of the effects of covariates on the survival status of child is made with the help of relative risks $e^{\beta}$ of each covariate.

Table 1 depicts the Omnibus test for model coefficients of in 6 steps of the analysis. It has been confirmed from the score tests which are statistically significant for all possible 6 models and thus overall coefficients of the models up to 6 steps are significant. Again, chi-square tests for change of next step from previous step are also found to be statistically significant and hence there is some improvement of the model from its previous model. Therefore, the model obtained at $6^{\text {th }}$ step is the best model fitted to the present data. Further, the improvement of a particular block from the previous block is also significant statistically up to $6^{\text {th }}$ step. In summary, it is said that the model obtained at the $6^{\text {th }}$ step is the best model in all aspects.

Table 2 shows the Cox's regression analysis by stepwise method (Wald's forward). In the table, estimated coefficients $(\beta)$ of covariates, standard error of $\beta$ estimates (SE), Wald's test statistic values, $p$ values of Wald's test, relative risks of covariates on child survival $\left(e^{\beta}\right)$ and $95 \%$ confidence interval of relative risks are shown. In first step, the DBF (duration 
Table 1: Omnibus Tests of Model Coefficients for Cox's Proportional Hazard Regression

\begin{tabular}{|c|c|c|c|c|c|c|c|c|c|c|}
\hline \multirow{2}{*}{ Step } & \multirow{2}{*}{$\begin{array}{c}-2 \text { Log } \\
\text { Likeliho od }\end{array}$} & \multicolumn{3}{|c|}{ Overall (score) } & \multicolumn{2}{c|}{ Change from previous step } & \multicolumn{3}{c|}{ Change from previous block } \\
\cline { 3 - 11 } & Chi-square & df & P-value & Chi-square & df & P-value & Chi-square & df & P-value \\
\hline \hline 1 & 402.163 & 195.617 & 1 & $<0.001$ & 69.706 & 1 & $<0.001$ & 69.706 & 1 & $<0.001$ \\
\hline 2 & 359.144 & 251.174 & 2 & $<0.001$ & 43.018 & 1 & $<0.001$ & 112.724 & 2 & $<0.001$ \\
\hline 3 & 350.393 & 291.080 & 3 & $<0.001$ & 8.752 & 1 & 0.003 & 121.476 & 3 & $<0.001$ \\
\hline 4 & 340.966 & 304.156 & 4 & $<0.001$ & 9.427 & 1 & 0.002 & 130.902 & 4 & $<0.001$ \\
\hline 5 & 326.259 & 317.242 & 5 & $<0.001$ & 14.707 & 1 & $<0.001$ & 145.609 & 5 & $<0.001$ \\
\hline 6 & 315.155 & 325.667 & 6 & $<0.001$ & 11.104 & 1 & 0.001 & 156.713 & 6 & $<0.001$ \\
\hline
\end{tabular}

of breastfeeding) is entered in the model and selected as the most important covariate out of 8 variables. In the second step, in addition to DBF, DPT is entered in the model and subsequently at the $6^{\text {th }}$ step, the six covariates viz., TTF (type of toilet facility), NHM (number of household members), CMT (use contraceptive), DBF (duration of breastfeeding), DPT and ING (ideal number of girls) are entered in the model and these six covariates comprise the best set of the covariates which can explained the survival status of child. These six covariates have negative relationship with the survival status of child.

The hazard ratio or relative risk of the covariate TTF is 0.159 and it is as little as 0.077 or as much as 0.330 with $95 \%$ confidence. It means that the hazard rate of child reduces by 15.9 percent in households with sanitary latrine as compared with the households

Table 2: Cox's Regression Analysis of Survival Time of Child by Stepwise Method

\begin{tabular}{|c|c|c|c|c|c|c|c|c|}
\hline \multirow{2}{*}{ Step } & \multirow{2}{*}{ Covariates } & \multirow{2}{*}{ B } & \multirow{2}{*}{ SE } & \multirow{2}{*}{ Wald } & \multirow{2}{*}{ P-value } & \multirow{2}{*}{$\operatorname{Exp}(B)$} & \multicolumn{2}{|c|}{$95.0 \% \mathrm{Cl}$ for $\operatorname{Exp}(\mathrm{B})$} \\
\hline & & & & & & & Lower & Upper \\
\hline 1 & DBF & -3.170 & 0.334 & 90.060 & $<0.001$ & 0.042 & 0.022 & 0.081 \\
\hline \multirow{2}{*}{2} & DBF & -2.879 & 0.338 & 72.424 & $<0.001$ & 0.056 & 0.029 & 0.109 \\
\hline & DPT & -2.228 & 0.382 & 34.051 & $<0.001$ & 0.108 & 0.051 & 0.228 \\
\hline \multirow{3}{*}{3} & TTF & -1.085 & 0.361 & 9.018 & 0.003 & 0.338 & 0.166 & 0.686 \\
\hline & DBF & -2.524 & 0.365 & 47.935 & $<0.001$ & 0.080 & 0.039 & 0.164 \\
\hline & DPT & -1.915 & 0.404 & 22.414 & $<0.001$ & 0.147 & 0.067 & 0.326 \\
\hline \multirow{4}{*}{4} & TTF & -1.173 & 0.363 & 10.433 & 0.001 & 0.309 & 0.152 & 0.630 \\
\hline & CMT & -1.228 & 0.438 & 7.868 & 0.005 & 0.293 & 0.124 & 0.691 \\
\hline & DBF & -2.233 & 0.376 & 35.263 & $<0.001$ & 0.107 & 0.051 & 0.224 \\
\hline & DPT & -1.617 & 0.415 & 15.220 & $<0.001$ & 0.198 & 0.088 & 0.447 \\
\hline \multirow{5}{*}{5} & TTF & -1.573 & 0.366 & 18.479 & $<0.001$ & 0.207 & 0.101 & 0.425 \\
\hline & NHM & -0.297 & 0.092 & 10.443 & 0.001 & 0.743 & 0.620 & 0.890 \\
\hline & CMT & -1.461 & 0.436 & 11.216 & 0.001 & 0.232 & 0.099 & 0.546 \\
\hline & DBF & -2.162 & 0.370 & 34.210 & $<0.001$ & 0.115 & 0.056 & 0.237 \\
\hline & DPT & -1.731 & 0.406 & 18.180 & $<0.001$ & 0.177 & 0.080 & 0.393 \\
\hline \multirow{6}{*}{6} & TTF & -1.836 & 0.371 & 24.542 & $<0.001$ & 0.159 & 0.077 & 0.330 \\
\hline & NHM & -0.339 & 0.080 & 17.977 & $<0.001$ & 0.713 & 0.609 & 0.834 \\
\hline & CMT & -1.398 & 0.447 & 9.798 & 0.002 & 0.247 & 0.103 & 0.593 \\
\hline & DBF & -2.342 & 0.367 & 40.780 & $<0.001$ & 0.096 & 0.047 & 0.197 \\
\hline & DPT & -1.920 & 0.404 & 22.609 & $<0.001$ & 0.147 & 0.066 & 0.323 \\
\hline & ING & -0.678 & 0.209 & 10.481 & 0.001 & 0.508 & 0.337 & 0.765 \\
\hline
\end{tabular}


without sanitary latrine, at any time and a reduction in the hazard rate of between 70.5 percent and 76.8 percent is consistent with the data.

Table 3: Mean of Covariates

\begin{tabular}{|c|c|}
\hline Covariates & Mean \\
\hline \hline TTF & 0.91 \\
\hline NHM & 6.13 \\
\hline TCB & 2.80 \\
\hline CMT & 0.55 \\
\hline DBF & 0.95 \\
\hline DPT & 0.76 \\
\hline PAR & 2.54 \\
\hline ING & 1.30 \\
\hline
\end{tabular}

In favour of this finding, Roth \& Kurup (1989) [22] suggest that good public sanitation systems may constitute a more important preventive aspect of child survival. In the latter study of Kabir \& Amin (1993) [23] in Bangladesh also highlights that the households with sanitary latrines have low risks of child mortality. The similar finding is also reported by Pandey et al. (1998) [5] on their study of infant and child mortality in India, a subject report of NFHS-2 and they have mentioned that access to a flush or pit toilet households have substantial and often statistically significant adjusted effects on infant and child mortality. The adjusted effect on mortality of household access to a flush or pittoilet is strongest for the neonatal period and becomes weaker at later ages. The adjusted effect tends to be significant in states with relatively high levels of neonatal mortality: Uttar Pradesh, Orissa, West Bengal and Assam. This pattern suggests that the lack of access to a flush or pit toilet is associated with increased risk of neonatal tetanus. As highlighted by Klaauw and Wang (2004) [12], access to sanitation facilities i.e., access to toilet facility can reduce under-five mortality rate significantly in rural areas of India as a whole. In urban Kenya, access to modern sanitation facilities (flush toilets) reduces diarrhea prevalence in urban areas and ultimately reduces the child mortality (Mutunga (2004) [30]). In a study of Balk et al. (2005) [26], the principal component analysis is used to combine the correlated variables which influence on mortality. From this analysis it is found that the mortality is correlated positively with the complete lack of toilet facilities and negatively with access to flush toilets. It is also suggested by Vos et al. (2005) [27] that the availability of better sanitation will decrease the probability of infant death since better sanitation and drinking water access by thehousehold should positively improve hygienic and health conditions for all members. On the other hand, Baker (1999) [24] and Rutstein (2000) [11], in contrary to above findings, observe that access of pit latrine does not have a significant effect on child mortality in the country.

The hazard ratio of NHM is 0.713 with $95 \%$ confidence interval (0.609-0.839) and it suggests that the total hazard rate of child reduces by 71.3 percent when one member is increased at any time in the existing number of family members. And, the hazard ratio as low as 0.609 or as high as 0.839 is consistent with the observed data at $5 \%$ level of significance. Many researchers like Srivastava (1994) [31] and Kabagenyl \& Rutaramwa (2013) [32] also suggest the same finding and concluded that the effect of family size on child mortality is statistically and substantially strong.

The estimated hazard ratio of CMT (use contraceptive) by mother is 0.247 with $95 \%$ confidence interval (0.103-0.593) and it infers that risk of child death is 24.7 less in those children born to mother using contraceptive than those children born to mother not using contraceptive, throughout the study period. And the hazard ratio between 0.103 and 0.593 is consistent with the observed data at 0.05 level of significance.

The present finding is in line of the findings of Tsui \& Creanga (2009) [33] and Saha \& Soest (2013) [34]. Mensch in his study on the effect of child mortality on contraceptive use and fertility in Colombia, Costa Rica and Korea, suggests that contraceptive use by women tends to reduce child mortality. Saha \& Soest (2013) also express that complete contraceptive use could reduce infant mortality of birth order two and higher by 7.9 percent. The net effect of complete contraceptive use on the total infant mortality rate is small, because the favorable effect on higher order births is partly offset by the rise in the proportion of high-risk first births.

The hazard rate of duration of breastfeeding (DBF) is 0.096 with $95 \%$ confidence interval $(0.047-0.197)$. It suggests that child reduces by 9.6 percent when duration of breastfeeding is less than 6 months and it may be as little as 4.7 percent or as much as 19.7 percent with $95 \%$ confidence with the study data, at any time, given other covariates held constant. 
Table 4: Survival Function Constructed by Cox's Hazard Regression Model

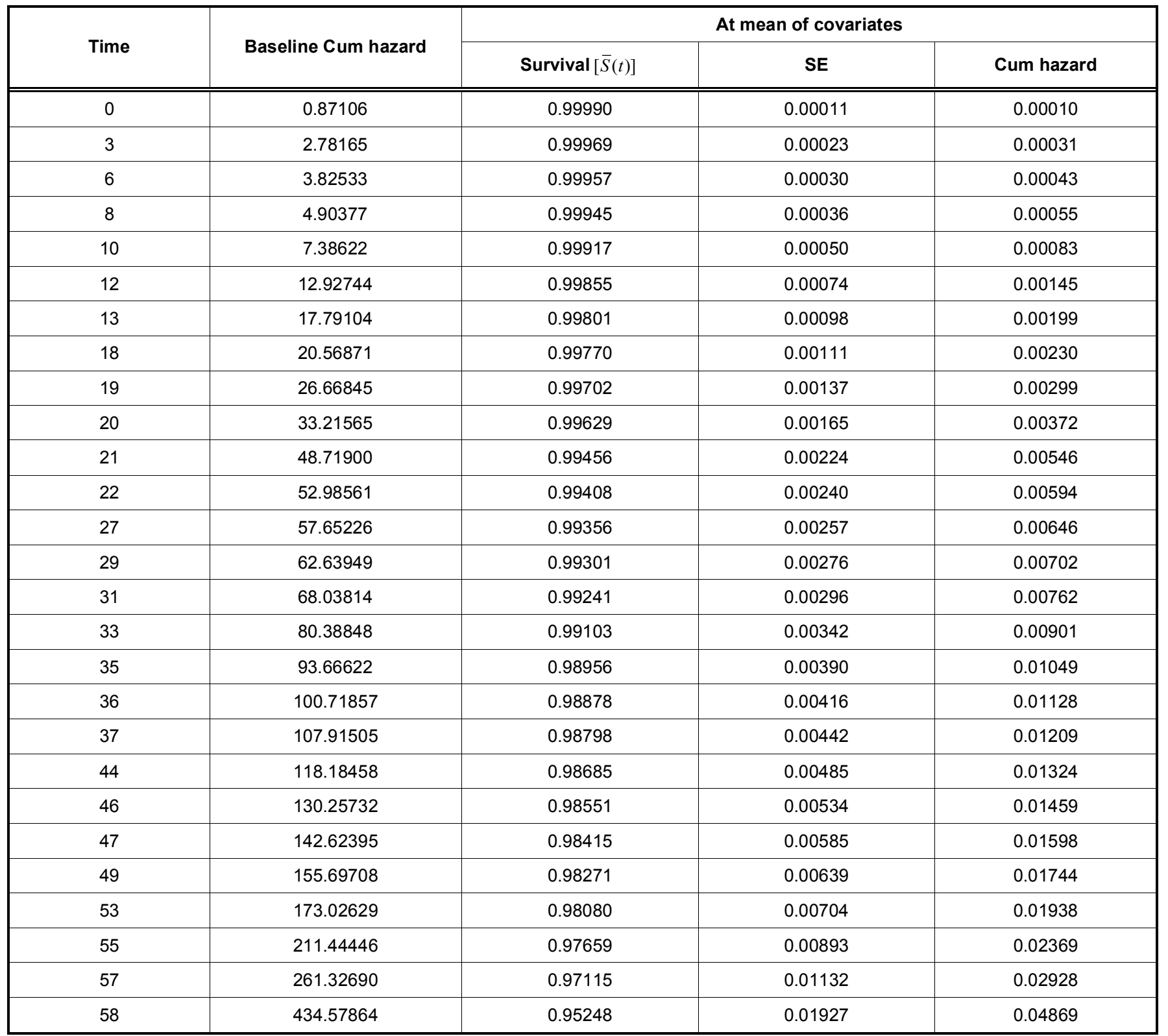

The present statement is in collusion with elsewhere findings of Palloni \& Millman (1985) [35], Fauveau et al. (1990) [36], Sandiford et al. (1991) [18], UNICEF (1997) [37], Pandey et al. (1998) [5] and Claeson et al. (1999) [19], as they highlight that breastfeeding promotion might be expected to have its largest effect on infant mortality. And it is also reported by Bhuyan (2000) [10] that duration of breastfeeding and age at marriage of mother have some influence in reducingmortality level of children. Rutstein (2000) [10] suggests that an increase in the duration of breastfeeding entails with a fall in post-neonatal mortality. A rise in the percentage of children aged 7-9 months who were both breastfed and getting solid foods is associated with decrease in both post natal and infant mortality rates. Biswas et al. (2000) [6] also report in their study on impact of some biosocial variables on infant and child mortality that breastfeeding appears to be prime factor influencing infant during second year (12-23 months) and early child (24-59 months). Further they suggest breastfeeding more than one year appears to have greatest potential for reducing infant and childhood mortality.

Further, Mahy (2003) [21] indicates that the vertical transmission of HIV occurs in approximately 32 percent of births to HIV infected mothers in countries where breastfeeding is prevalent and it will directly affect on childhood mortality too. 
The DPT(diphtheria, pertussis, tetanus) vaccine given to mothers during pregnancy has likely to have $14.7 \%$ less chance of their child death as compared with others as evident by hazard ratio 0.147 with $95 \%$ confidence interval (0.066-0.323) keeping effects of other covariates constant.

The estimated hazard ratio for ING (ideal number of girls) is 0.508 with $95 \%$ confidence interval (0.3370.765 ) and it interprets that the hazard rate reduces by $50.8 \%$ for every one increase in ideal number of girls desired by parents and a decrease in the hazard rate of between 33.7 and 76.5 percent is consistent with the data.
Table 5 shows the life table of children under study. One child is death before reaching one month after birth and the estimated survival chances of children within one month is 0.99473 . The two children are died in between first and third months after birth and their survival chances at that time is 0.99717 . Another one child is died at $6^{\text {th }}$ month of birth and its survival chance is 0.94629. Similarly, the survival chances of the children on $8^{\text {th }}, 10^{\text {th }}$ and $12^{\text {th }}$ months after birth are estimated at $0.99956,0.85694$ and 0.72724 respectively. At the end of the table, two children are died and the survival chance of each of them on $58^{\text {th }}$ month is 0.77214 . Further, it is observed that the survival chances of children are sometimes increase in some months and then reduces in other months i.e.,

Table 5: Life Table of Children Under Study

\begin{tabular}{|c|c|c|c|c|c|}
\hline $\begin{array}{l}\text { Age of child } \\
\text { (month) }\end{array}$ & No. of deaths & Cumulative number of deaths & Number of alive & $\bar{S}(t)$ & $S(t)$ \\
\hline 0 & 1 & 1 & 835 & 0.99990 & 0.99473 \\
\hline 3 & 2 & 3 & 833 & 0.99969 & 0.99717 \\
\hline 6 & 1 & 4 & 832 & 0.99957 & 0.94629 \\
\hline 8 & 1 & 5 & 831 & 0.99945 & 0.99956 \\
\hline 10 & 2 & 7 & 829 & 0.99917 & 0.85694 \\
\hline 12 & 3 & 10 & 826 & 0.99855 & 0.72724 \\
\hline 13 & 2 & 12 & 824 & 0.99801 & 0.88604 \\
\hline 18 & 1 & 13 & 823 & 0.99770 & 0.92966 \\
\hline 19 & 2 & 15 & 821 & 0.99702 & 0.82320 \\
\hline 20 & 2 & 17 & 819 & 0.99629 & 0.85390 \\
\hline 21 & 4 & 21 & 815 & 0.99456 & 0.81115 \\
\hline 22 & 1 & 22 & 814 & 0.99408 & 0.98098 \\
\hline 27 & 1 & 23 & 813 & 0.99356 & 0.88597 \\
\hline 29 & 1 & 24 & 812 & 0.99301 & 0.97971 \\
\hline 31 & 1 & 25 & 811 & 0.99241 & 0.92344 \\
\hline 33 & 2 & 27 & 809 & 0.99103 & 0.93884 \\
\hline 35 & 2 & 29 & 807 & 0.98956 & 0.91925 \\
\hline 36 & 1 & 30 & 806 & 0.98878 & 0.97568 \\
\hline 37 & 1 & 31 & 805 & 0.98798 & 0.98896 \\
\hline 44 & 1 & 32 & 804 & 0.98685 & 0.86233 \\
\hline 46 & 1 & 33 & 803 & 0.98551 & 0.86999 \\
\hline 47 & 1 & 34 & 802 & 0.98415 & 0.97254 \\
\hline 49 & 1 & 35 & 801 & 0.98271 & 0.99190 \\
\hline 53 & 1 & 36 & 800 & 0.98080 & 0.86962 \\
\hline 55 & 1 & 37 & 799 & 0.97659 & 0.99172 \\
\hline 57 & 1 & 38 & 798 & 0.97115 & 0.87707 \\
\hline 58 & 2 & 40 & 796 & 0.95248 & 0.77214 \\
\hline
\end{tabular}


there is no uniform trend of either decrease or increase of survival chances of children with respect to time.

\section{RECOMMENDATIONS}

The present findings provide information of health planners and managers responsible for programmes to reduce child mortality. Encouraging effective immunization programmes to children such as immunization of DPT will greatly enhance the survival of children. Avoiding high-order births and extending the duration of breastfeeding will also substantially enhance survival chances of children during the first five years of life. Awareness of Family Planning Programme to couples to maintain less number of children will also help to reduce child mortality and no sex preference should be encouraged. Improving toilet facility of household will greatly influence to reduce child mortality and hence it should be provided to each and every household.

\section{REFERENCES}

[1] WHO. Child survival and health. World Heath Organisation, Geneva, Switzerland 2005.

[2] Mosley WH, Chen LC. An analytical frme work for the study of child survival in developing countries. Population \& Dev Rev 1984; 10(Suppl): 25-45. http://dx.doi.org/10.2307/2807954

[3] Hobcraft JN, McDonald JW, SO. R. Demographic determinants of infant and early child mortality: a comparative ananlysis. Population Studies 1985; 39(3): 36385.

\section{http://dx.doi.org/10.1080/0032472031000141576}

[4] Majumder AK, May M, Pant PD. Infant and child mortality differentials in Bangladesh: are they changing. $\mathrm{J}$ biosoc Sci 1997; 29: 385-99. http://dx.doi.org/10.1017/S0021932097003854

[5] Pandey A, Choe MK, Luther NY, Sahu D, Chand J. Infant and child mortality in India. NFHS Subject Reports, No.11, Dec 1998, IIPS, Mumbai India 1998.

[6] Biswas SC, Rahman IK, Malaque MA. Impact of some biosocial variables on infant and child mortality. Demography India 2000; 29(2): 211-21.

[7] Syamala TS. Relationship Between Socio Demographic Factors and child survival: evidences from Goa, India. J Hum Ecol 2004; 16(2): 141-5.

[8] Bahlotra S, Soest Av. Birth spacing and neonatal mortality in India: dynamics, frailty and fecundity. Discussion Paper No 2005-06, RAND Labor and Popualtion 2005

[9] National Family Health Survey (NFHS-3) 2005-06 India Volume I. Mumbai, India: IIPS under Ministry of Health and Family Welfare, Govt. of India 2008.

[10] Bhuyan KC. Differential in child mortality by fertility in NorthEastern Libya. Sankhya 2000; 62(B(2)): 317-26.

[11] Rutstein SO. Factors associated with trends in infant and child mortality in developing countries during the 1990s. Bulletin of WHO 2000; 78: 1256-70.

[12] Klaauw BVD, Wang L. Child mortality in rural India. Discussion Paper of World Bank, Washington DC, USA 2004.
Adair T. Child mortality in indonesia's mega-urban regions: measurement, analysis of differentials, and policy implications. Paper presented at: 12th Biennial Conference Population and Society: issues, research, policy on 15-17 Sep2004; Canberra, Australia: Canberra, Australian Bureau of Statistics 2004.

[14] IIPS. National Family Health Survey (NFHS--3): 2005-06. Mumbai, India: IIPS, 2007 September.

[15] WHO. Infant and child mortality in the third world 1983.

[16] WHO. Global immunization vision and strategy. Geneva: WHO, 2010, 25 November.

[17] Fauvcau V, Wotyniak B, Chakraborty J, Sarder AM, Briend A The effect of maternal and child health and family planning services on mortality: is prevention enough? BMJ 1990; 301 : 103-7.

http://dx.doi.org/10.1136/bmj.301.6743.103

[18] Sandiford P, Morale P, Gorter A, Coyle E, Smith D. Why do child mortality rates fall? An analysis of the Nicaraguan experience. Am J Public Health 1991; 81(1): 30-7. http://dx.doi.org/10.2105/AJPH.81.1.30

[19] Claeson M, Bos E, Pathmanathan I. Reducing Child Mortality in India: keeping up the pace 1999.

[20] Griffiths P, Hinde A, Mathews Z. Infant and child mortality in three cuturally contrasting states of India. J Biosoc Sci 2001 33: 603-22. http://dx.doi.org/10.1017/S0021932001006034

Mahy M. Measuring child mortality in AIDS affected countries. Paper presented at: Workshop on HIVIAIDS and adult mortality in developing countries: Department of Economic and Social Affairs, UNO, on 8-13 Sep 2003, NY, USA 2003.

[22] Roth E, Kurup B. Child mortality levels and survival patterns from Southern Sudan. Demography India 1989; J8(J989): 139-46.

[23] Kabir M, Amin R. Factors influencing child mortality in Bangladesh and their implications for the National Health Programme. Asia-Pacific Population Journal 1993; 8(3): 3146.

[24] Baker R. Differential in child mortality in Malawi. Social Networks Project Working Papers, No. 3, Spring 1999, University of Pennsylvania, USA 1999.

[25] Environmental Determinants of Child Mortality in Urban Kenya(P).pdf.

[26] Balk D, Storeygard A, Levy M, Gaskell J, Sharma M, Flor R. Child hunger in the developing world: analysis of environmental and social correlates. Food Policy 2005; 30: 584-611. http://dx.doi.org/10.1016/j.foodpol.2005.10.007

[27] Vos R, Cuesta J, Leon M, Lucio R, Orsero J. Reaching the Millennium Development Goal for child mortality: improving equity and efficiency in Ecuador's health budget. The Hague and Quito 2005.

[28] Claeson M, Bos ER, Mawji T, Pathmanathan I. Reduced child mortality in India in new millennium. Bulletin of WHO 2000; 78: 1192-9.

[29] Cox DR. Regression Models and Life-Tables. J R Statist Soc B 1972; 34(2): 187-220.

[30] Mutunga CJ. Environmental determinants of child mortality in Urban Kenya. Abdus Salam ICTP; Trieste, Italy 2004 April.

[31] Srivastava JN. Impact of child mortality on family size desires and familiy planning practice among white-collar workers. $J$ Fam Welfare 1994; 40(2): 19-26.

[32] Kabagenyl A, Rutaremwa G. The effect of household characteristics on child mortality in Uganda. Am J Socio Res 2013; 3(1): 1-5.

[33] Tsui AO, Creanga AA. Does contraceptive use reduce neonatal and infant mortality? Findings from a multi-country 
analysis. Population, Family \& Reproductive Health Development. Johns Hopkins Bloomberg School of Public Health 2009.

[34] Saha UR, Soest AV. Contraceptive use, birth spacing and child survival in Matlab, Bangladesh. Studies of Fam Planning 2013; 44(1): 45-66. http://dx.doi.org/10.1111/j.1728-4465.2013.00343.x

[35] Palloni A, Millman S. Effects of interbirth intervals and breastfeeding on infant and early childhood mortality. CDE
Working Paper 85-11. Meeting of the Population Association of America; Boston, USA 1985.

[36] Fauveau V, Wotyniak B, Chakraborty J, Sarder AM, Briend A. The effect of maternal and child health and family planning services on mortality: is prevention enough? BMJ 1990; 301: 103-7.

http://dx.doi.org/10.1136/bmj.301.6743.103

[37] BASICS. The recent evoluation of child mortality in Developing world 1997. 